\title{
New Combination of Ultraviolet Absorbers in an Oily Emollient Increases Sunscreen Efficacy and Photostability
}

Florence L'Alloret • Didier Candau • Sophie Seité • Marie-Jocelyne Pygmalion • Laetitia Ruiz •

Martin Josso $\cdot$ Hélène Meaudre $\cdot$ Lydie Gauchet $\cdot$ Ana-Maria Pena $\cdot$ Anne Colonna

To view enhanced content go to www.dermtherapy-open.com

Received: February 3, 2012 / Published online: April 17, 2012

(c) The Author(s) 2012. This article is published with open access at Springerlink.com

\section{ABSTRACT}

Introduction: It is now recognized that to adequately protect skin from sun damage, sunscreens require a photostable combination of ultraviolet (UV) filters with a suitable level of UVA protection. The total amount of UV filters should be as low as possible to avoid adverse skin reactions, potential environmental impact, and to ensure acceptable texture for better application and usage.

F. L'Alloret - D. Candau - M. Josso - H. Meaudre · A. Colonna

L'Oréal Research and Innovation, Chevilly Larue, France

S. Seité $(\varangle)$

La Roche-Posay Pharmaceutical Laboratories, 110 Avenue Henri Barbusse, 92600 Asnières, France e-mail: sophie.seite@loreal.com

M.-J. Pygmalion · L. Ruiz · L. Gauchet · A.-M. Pena L'Oréal Research and Innovation, Aulnay sous-bois, France

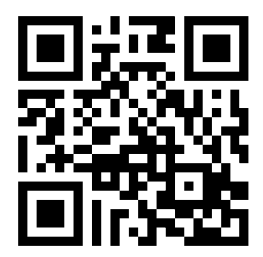

Enhanced content for this article is available on the journal web site: www.dermtherapy-open.com
Methods: A synergistic combination of UV filters was selected to obtain a high sun protection factor (SPF) and UVA protection factor (UVA-PF). An oily vehicle was then added to the formula to improve the solubility and the photostability of the lipophilic UV filters.

Results: The combination of filters, i.e., terephthalylidene dicamphor sulfonic acid (TDSA), bis-ethylhexyloxyphenol methoxyphenyl triazin (BEMT), and butyl methoxy dibenzoyl methane (BMDM), obtained an elevated SPF as well as a high UVA-PF. Isopropyl lauroyl sarcosinate (ILS), a derivative of a natural amino acid (sarcosine, also known as $\mathrm{N}$-methylglycine) was introduced in this formulation in order to dissolve the oil-soluble UV absorbers and to photostabilize BMDM. The new sunscreen formulation obtained with this combination is photostable and contains a reduced amount of UV filters compared to other sunscreens with the same level of efficacy.

Conclusion: This report described the steps resulting in the formulation of a new combination of UV filters in an oily emollient, which presents a high UVA-PF (UVA-PF $=38$ ) and a SPF 50+, is photostable, and offers good protection against UV-induced biological damage. 
Keywords: Isopropyl lauroyl sarcosinate; Photoprotection factors; Photostability; Sunscreen; Sun protection factor; UV absorbers; UV filters; UV protection

\section{INTRODUCTION}

Most people use sunscreens to avoid sunburn, whilst the more educated of those also know that sunscreen can protect their skin from premature aging and cancer [1]. However, the use of sunscreens is far from what could be expected [2]. The reason frequently conveyed by consumers for avoiding sunscreen use is that they are too greasy and leave an unpleasant feeling on the skin. If they are used, they are not applied in sufficient quantity, perhaps due to their unpleasant texture, but also a lack of information regarding their correct usage [3]. Furthermore, skin reactions to sunscreen agents may be an issue. Contact dermatitis and photocontact sensitization can be a concern for some people [4,5], although it is mostly subjective irritation or discomfort that is the actual problem [6]. Finally, a rising objection to sunscreen use is the potential risk to the environment, particularly for aquatic organisms. However, these concerns may be addressed by lowering the concentration of ultraviolet (UV) filters in the formulations. This has to be achieved without compromising efficacy, which is a difficult challenge.

Increasing evidence of the damaging effects of UVA impel sunscreen formulators to use new UVA filters [7]. Consumers, as well as the European Commission (EC), have also requested greater and wider protection against solar UVA radiation [8]. To meet these demands, researchers developed UVA filters [9]. One of the first filters available was butyl methoxy dibenzoyl methane (BMDM).
Unfortunately, it has been shown that this molecule loses part of its absorbance under UV-exposure; it is photo-labile or photounstable [10].

For many years, BMDM was the only longwave UVA filter (maximum wavelength = $357 \mathrm{~nm}$ ) allowed in Europe and the US. However, photostability has been, and remains, a primary focus for formulators. Photostability is obtained by removing certain UV filters and excipients known to be deleterious to BMDM, and by including ingredients known to improve its photostability [11].

A further challenge for the sunscreen formulator is to reduce the total amount of chemical filters without compromising the efficacy. Therefore, the authors set out to find a synergetic combination of UV filters in terms of sun protection factor (SPF) and UVA protection factor (UVA-PF). In addition, an oily vehicle, which could improve the solubilization of the lipophilic UV filters, as well as ensuring BMDM photostability, was added to this combination.

The photostability, SPF, UVA-PF, and the in vivo efficacy in the prevention of photoreactions observed after yearly sun exposure [e.g., polymorphous light eruption (PLE)] were evaluated for this new complete sunscreen formulation.

\section{MATERIALS AND METHODS}

\section{Sunscreen Products}

The list of active materials [trade names, International Nomenclature of Cosmetic Ingredients (INCI) names, and maximum absorption for the UV filters] is given in Table 1. The first part of the work was performed with simplex formulas, detailed in 
Table 2, and the second part with complete SPF $50+$ formulations, listed in Table 3.

\section{Photostability}

To evaluate the photostability of the BMDM, the residual concentration of $2 \%$ of BMDM was introduced in the simplex oil in water emulsion with and without bis-ethylhexyloxyphenol methoxyphenyl triazin (BEMT) and isopropyl lauroyl sarcosinate (ILS), a derivative of a natural amino acid (sarcosine, also known as $\mathrm{N}$-methylglycine) (Sunscreens F, G, and H). This was measured by high-performance liquid chromatography (HPLC) after exposure to UV solar simulated radiation (SSR) with an Atlas Suntest ${ }^{\circledR}$ CPS (Atlas, Chicago, IL, USA), as previously described $[12,13]$. The duration of

Table 1 Characteristics of ultraviolet filters

\begin{tabular}{llll}
\hline Trade name & Manufacturer & Abbreviation & $\begin{array}{l}\text { Wavelength at which } \\
\text { maximum absorption } \\
\text { occurs (nm) }\end{array}$ \\
\hline Uvinul $^{\circledR} 539$ & BASF Aktiengesellschaft, Ludwigshafen, Germany & OC & 303 \\
Mexoryl $^{\circledR}$ SX & L'Oréal, Paris, France & TDSA & 340 \\
Tinosorb $^{\circledR}$ S & BASF Aktiengesellschaft, Ludwigshafen, Germany & BEMT & $310-345$ \\
Mexoryl $^{\circledR}$ XL & L'Oréal, Paris, France & DTS & $304-340$ \\
Parsol $^{\circledR}$ 1789 & Givaudan Roure, New Jersey, USA & BMDM & 355 \\
Uvinul $^{\circledR}$ T 150 & BASF Aktiengesellschaft, Ludwigshafen, Germany & ET & 314 \\
Eldew $^{\circledR}$ SL-205 & Ajinomoto Co., Inc., Tokyo, Japan & ILS & - \\
\hline
\end{tabular}

$B E M T$ bis-ethylhexyoxyphenol methoxyphenyl triazin, BMDM butyl methoxydibenzoylmethane, DTS drometrizole trisiloxane, ET ethylhexyl triazon, ILS isopropyl $N$-lauroyl sarcosinate, $O C$ octocrylene, TDSA terephtalylidene dicamphor sufonic acid

Table 2 Composition of ultraviolet filters in Sunscreens A-H

\begin{tabular}{lllllllll}
\hline Chemical & \multicolumn{2}{l}{ Sunscreen } & & & & \\
\cline { 2 - 9 } & A & B & C & D & E & F & G & H \\
\hline TDSA (\%) & - & 8.11 & 2.46 & - & - & - & - & - \\
BEMT (\%) & 8.11 & - & 5.65 & - & 5.65 & - & 2.00 & - \\
BMDM (\%) & - & - & - & 1.96 & 0.76 & 2.00 & 2.00 & 2.00 \\
OC (\%) & - & - & - & 6.15 & 1.70 & - & - & - \\
Total amount of UV absorbers (\%) & 8.11 & 8.11 & 8.11 & 8.11 & 8.11 & 2.00 & 4.00 & 2.00 \\
Other components (ILS) (\%) & - & - & - & - & - & - & - & 10.00 \\
\hline
\end{tabular}

$B E M T$ bis-ethylhexyloxyphenol methoxyphenyl triazin, $B M D M$ butyl methoxy dibenzoyl methane, ILS isopropyl $N$-lauroyl sarcosinate, $O C$ octocrylene, TDSA terephthalylidene dicamphor sulfonic acid, $U V$ ultraviolet 
Table 3 Composition and characteristics of ultraviolet absorbers of SPF $50+$ Sunscreens I and J

\begin{tabular}{lll}
\hline Chemical & \multicolumn{2}{l}{ Sunscreen } \\
\cline { 2 - 3 } & $\mathbf{I}$ & $\mathbf{J}$ \\
\hline TDSA (\%) & 0.50 & 0.75 \\
BEMT (\%) & 0.50 & 2.00 \\
BMDM (\%) & 3.00 & 3.00 \\
Other UV filters (DTS, & 10.00 & 6.00 \\
$\quad$ & \\
OC, ET) (\%) & 14.00 & 11.75 \\
Total amount of UV & & \\
absorbers (\%) & No & Yes \\
ILS & $62.30 \pm 5.70$ & $69.60 \pm 4.50$ \\
SPF & $28.40 \pm 6.30$ & $38.40 \pm 9.50$ \\
UVA-PF &
\end{tabular}

$B E M T$ bis-ethylhexyloxyphenol methoxyphenyl triazin, $B M D M$ butyl methoxy dibenzoyl methane, DTS drometrizole trisiloxane, ET ethylhexyl triazon, ILS isopropyl $N$-lauroyl sarcosinate, $O C$ octocrylene, $S P F$ sun protection factor, TDSA terephthalylidene dicamphor sulfonic acid, UVA-PF ultraviolet A protection factor

exposure was calculated in order to deliver $18 \mathrm{~J} /$ $\mathrm{cm}^{2}$ of UVA (320-400 nm), which corresponds to the dose received during a $1 \mathrm{~h}$ exposure to the zenithal sun.

Furthermore, the absorption spectra of Sunscreens $\mathrm{H}$ and I were measured by UV spectrophotometry after exposure to SSR. Two different doses were given for each sunscreen: one dose was $45 \mathrm{~J} / \mathrm{cm}^{2}$ of UVA, the other was $75 \mathrm{~J} / \mathrm{cm}^{2}$ of UVA.

\section{SPF and UVA-PF}

The SPF was measured in vivo using the international SPF test method [14]. The UVA-PF, based on the persistent pigment darkening method, was measured using the method used in Japan [15]. The measurements were performed on the simplex emulsions $\mathrm{A}, \mathrm{B}$, and $\mathrm{C}$, as well as on the complete Sunscreens I and J
(Tables 2, 3). Ten subjects were used for each measurement.

\section{Protective Effect of Sunscreens Against Reactive Oxygen Species}

RealSkin $^{\mathrm{TM}} \quad$ (SkinEthnic laboratories, Lyon, France; $4 \mathrm{~cm}^{2}$ full-thickness skin equivalents) is a reconstructed skin (RS) model made of an epidermis and a living dermis. The generation of reactive oxygen species (ROS) was measured using the oxidant sensing probe, $2^{\prime}$, $7^{\prime}$-dichlorodihydrofluorescein diacetate (DCFH-DA), as previously described [16]. The RS was loaded by systemic application with freshly prepared DCFH-DA solution for $30 \mathrm{~min}$ before the application of sunscreen (A, B, C, D, and E), vehicle treatment, or nothing (the UVA control), which was applied $20 \mathrm{~min}$ prior to UVA exposure $\left(10 \mathrm{~J} / \mathrm{cm}^{2}, 320-400 \mathrm{~nm}\right)$. The UVA source was the $1.000 \mathrm{~W}$ Oriel ${ }^{\circledR}$ (Newport Corporation, CA, USA) solar simulator fitted with Schott AG optical filters UG11 and WG335. Immediately after UVA exposure, ROS generation was measured by spectrofluorimetry (excitation $485 \mathrm{~nm}$; emission $535 \mathrm{~nm}$; Tecan Spectrafluor Plus, Tecan Group Ltd, Switzerland). Each essay was performed on three different RS batches.

In addition, two-photon excited fluorescence (2PEF) was used to detect UV-induced ROS within RealSkin reconstructed epidermis (RE) with three-dimensional (3D) subcellular resolution. Multiphoton imaging was performed using a Zeiss LSM510 Meta microscope. 2PEF was excited by a femtosecond titanium-sapphire laser adjusted to $760 \mathrm{~nm}$, with typically $3 \mathrm{~mW}$ power at the sample. Samples were maintained between two cover slides and imaged by use of a $40 \times, 1.1$ numerical aperture, water-immersed, objective lens. The acquisition time per pixel was $2.05 \mu \mathrm{s}$. The authors recorded three $210 \times 210 \times 100 \mu \mathrm{m}^{3}$ 
z-stacks in every sample, with $1 \mu \mathrm{m}$ z-step and $0.6 \mu \mathrm{m}$ pixel size. Two-dimensional images were combined using Image software (W. Rasband, National Institutes of Health), and quantified (average intensity of fluorescence in each stack).

\section{In-Vivo Efficacy}

The efficacy of Sunscreen J in preventing cutaneous skin reactions associated with PLE, such as itching, burning, and urticarial papules, was evaluated during an "in use" test conducted under dermatological control during early summer in Cape Town, South Africa. No ethic committee approval was required for this test. Forty-one volunteers of Caucasian origin prone to PLE (at least three episodes, with one during the last summer), were recruited. After receiving instructions for use, the volunteers were asked to apply the product instead of their usual sunscreen at least once a day on their face, neck, and arms for 2 weeks. After swimming, volunteers had to re-apply the product. Volunteers were asked to report any cutaneous reactions during the test, which were confirmed by dermatologist examination, and to complete a questionnaire about the cosmetic qualities of the product.

\section{RESULTS}

The Association of Terephthalylidene

Dicamphor Sulfonic Acid and BEMT Has a Synergistic Effect in Terms of Protection Factors

With a total concentration of $8.11 \%$ of UV filters [Sunscreen C: $2.46 \%$ terephthalylidene dicamphor sulfonic acid (TDSA), 5.65\% BEMT] in a simplex emulsion, the authors obtained a

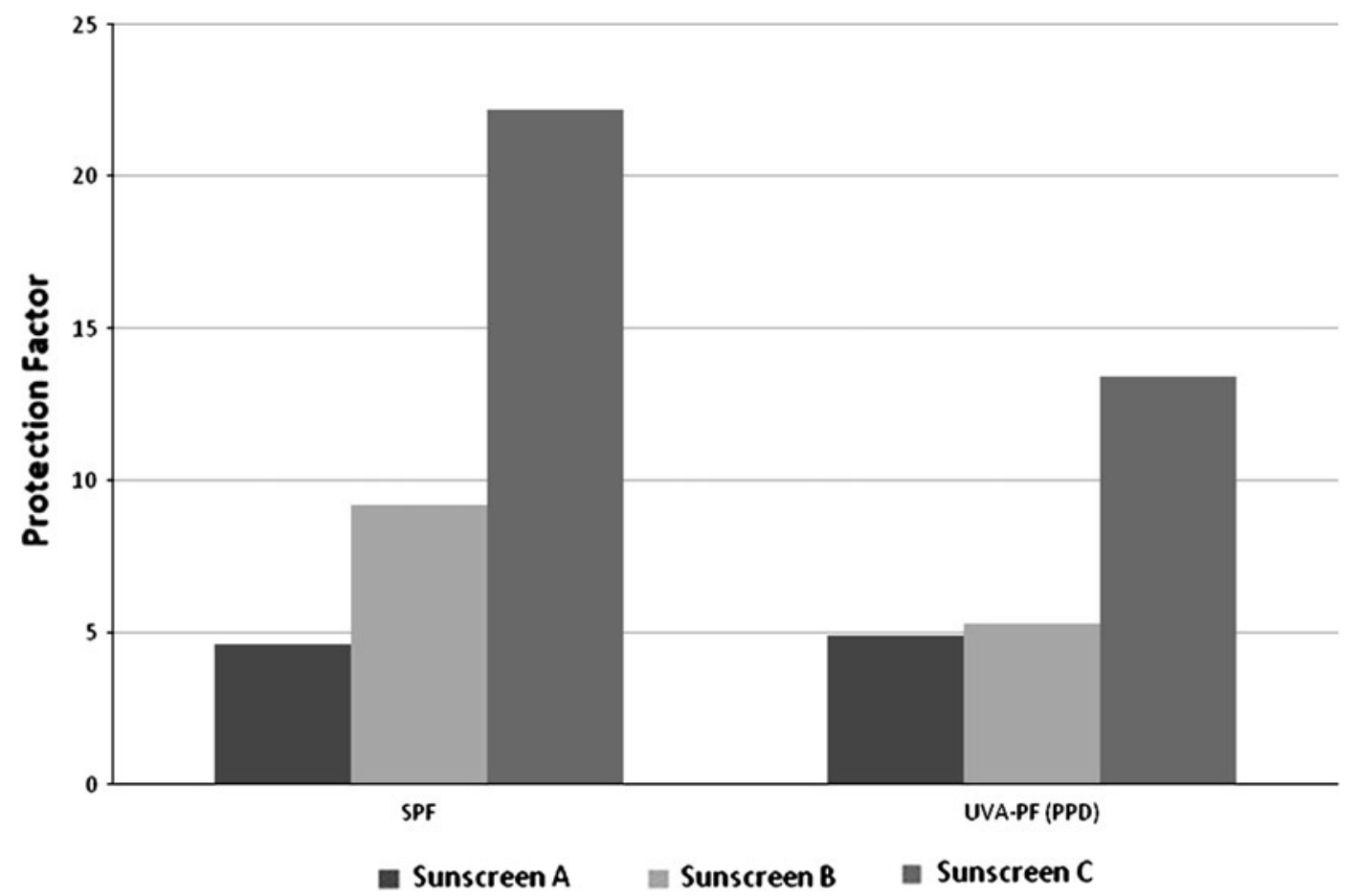

Fig. 1 SPF and UVA-PF comparison of sunscreens A, B, and $\mathrm{C}$ showing the synergistic effect of the association methoxyphenyl triazin, SPF sun protection factor, TDSA terephthalylidene dicamphor sulfonic acid, $U V A-P F$ ultraviolet A protection factor 
SPF value of $22.2 \pm 3.5$ and a UVA-PF of $13.4 \pm 1.5$ (Fig. 1). With $8.11 \%$ TDSA alone in the same emulsion (Sunscreen B), the SPF was $4.6 \pm 1.1$ and the UVA-PF $4.9 \pm 0.1$. With $8.11 \%$ BEMT alone in the same emulsion (Sunscreen A), the SPF was $9.2 \pm 2$ and the UVA-PF $5.3 \pm 0.9$ (Fig. 1).

\section{Association of TDSA and BEMT Offers Optimal Protection Against UVA-Induced Oxidative Stress}

Reactive oxygen species detection induced by UVA can be used to rate different sunscreen products from the most to the least effective in reducing oxidative stress. Figure 2 shows ROS detection induced by UVA, and suggests that Sunscreen $\mathrm{A} \approx \mathrm{B} \approx \mathrm{C}>\mathrm{E}>\mathrm{D}>$ vehicle $>$ UVA control. These sunscreen products all have the same total UV filter content (8.11\%, Table 2$)$.
Sunscreens C, D, and E have a similar UV absorption spectrum. To validate this result in the different epidermis layers, the authors performed a multiphoton acquisition test, as shown in Fig. 3.

\section{BMDM is Photostabilized by Association with BEMT or ILS}

After $1 \mathrm{~h}$ of exposure to SSR, which includes $18 \mathrm{~J} / \mathrm{cm}^{2}$ of total UVA, only $30 \%$ of the initial $2 \%$ of BMDM introduced in a simplex emulsion (Sunscreen F) was detected by HPLC. When $2 \%$ BEMT was added to this simplex emulsion (sunscreen G), a higher percentage of BMDM remained (approximately 78\%). Similarly, the addition of $10 \%$ ILS (which is not a filtering molecule) to this simplex emulsion (Sunscreen $\mathrm{H})$ allowed up to $75 \%$ photostabilization of BMDM, as shown on Fig. 4.

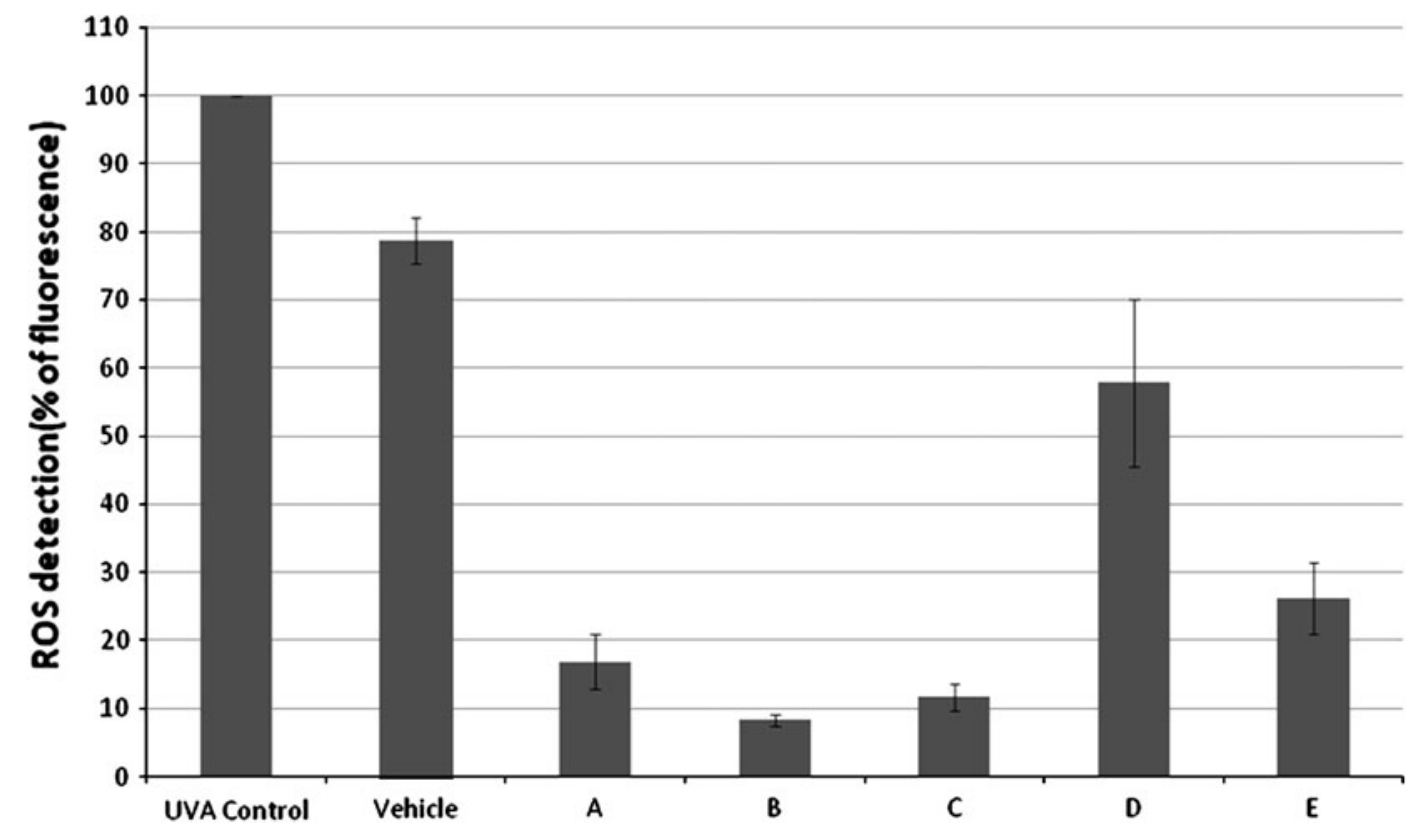

Fig. 2 Photoprotective efficacy of Sunscreens A, B, C, D, and $\mathrm{E}$ (same total UV filter content of $8.11 \%$ ) against UVA-induced ROS measured by spectrofluorimetry after DCFH-DA incubation on reconstructed human skin. Sunscreen C containing TDSA and BEMT offers good

protection against UVA-induced oxidative stress. BEMT bis-ethylhexyloxyphenol methoxyphenyl triazin, $D C F H-D A$ $2^{\prime}, 7^{\prime}$-dichlorodihydrofluorescein diacetate, $R O S$ reactive oxygen species, TDSA terephthalylidene dicamphor sulfonic acid 


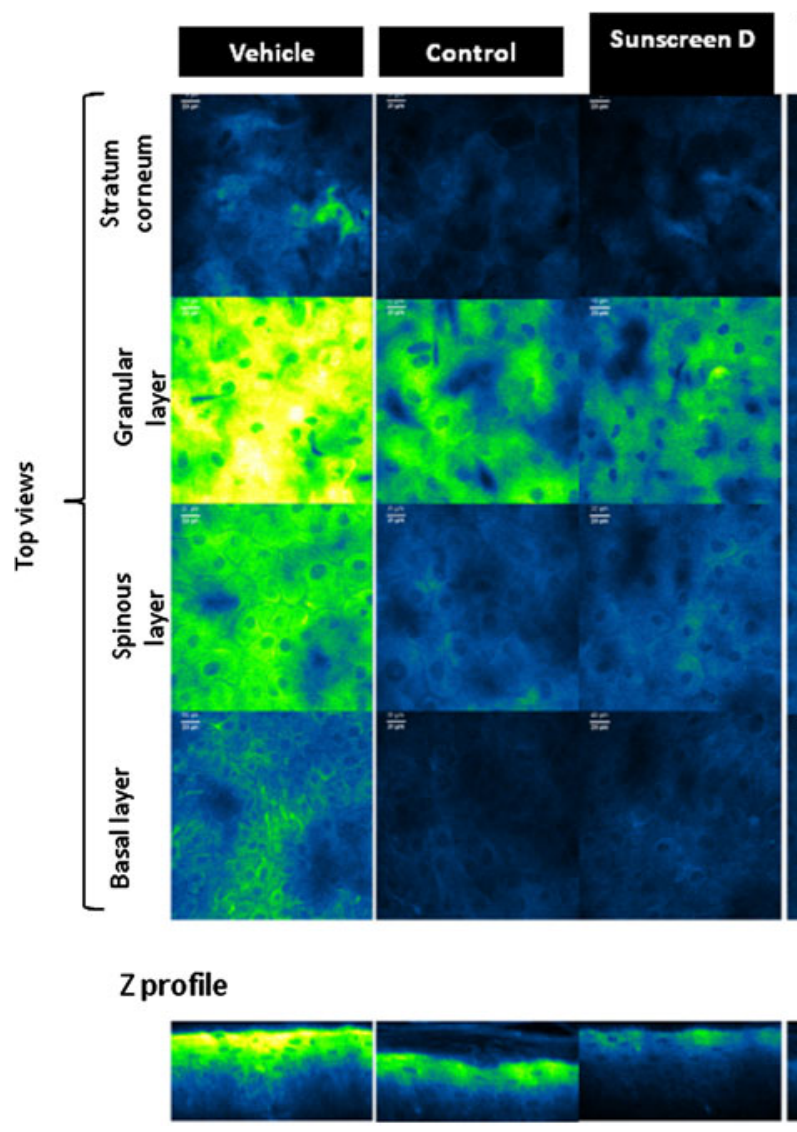

Fig. 3 Comparison of Sunscreens A, B, C, D, and E (same total UV filter content of $8.11 \%$ ) efficacy against the UVA-induced oxidative stress in the different reconstructed epidermis layers evaluated by two-photon excited fluorescence. Sunscreen C containing an association of TDSA and BEMT offers a good protection against

\section{SPF and UPA-PF of Sunscreens I and J}

The association of UV filters, including TDSA, BEMT, BMDM, and ILS, increased both the SPF and the UVA-PF even if the total amount of UV absorbers decreased from $14 \%$ in Sunscreen I (without ILS) to $11.75 \%$ in Sunscreen J (with ILS). The SPF and UVA-PF $( \pm S D)$ values are given at the bottom of Table 3 .

\section{Sunscreen J is Photostable}

Figure 5 shows the UV absorption spectra of Sunscreen J before and after SSR exposure,
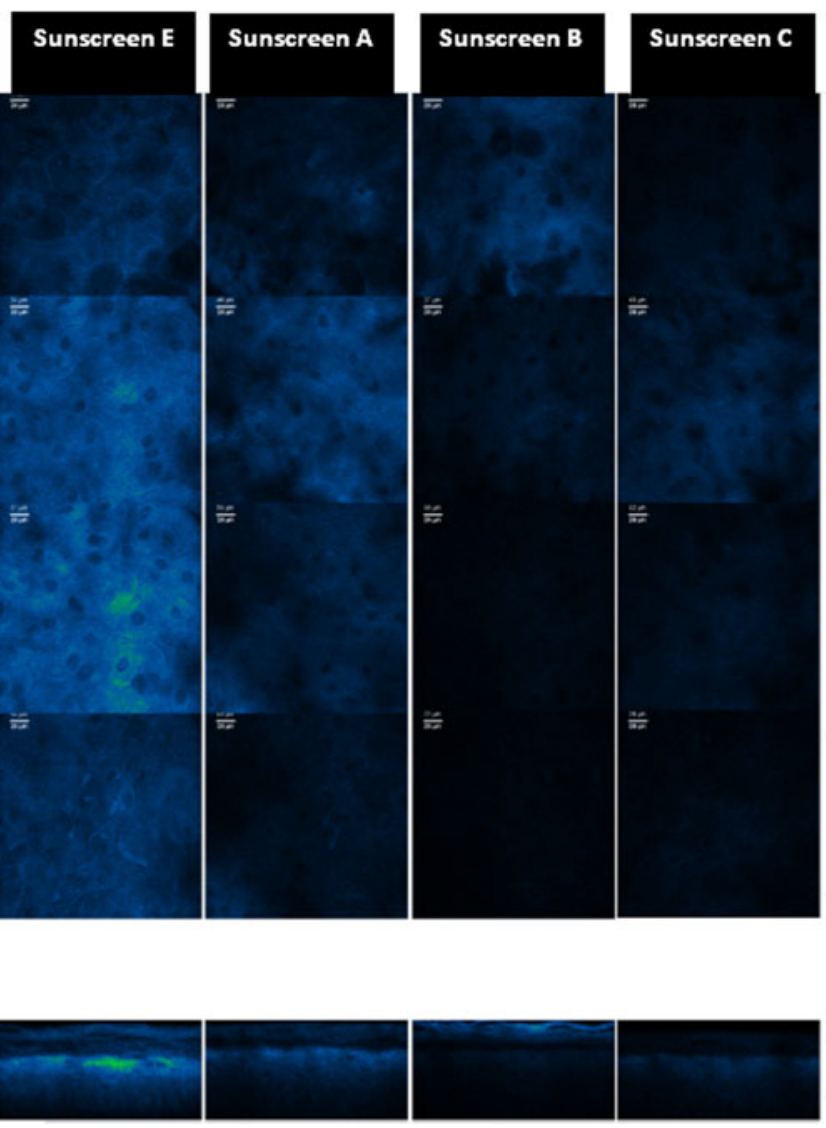

UVA-induced oxidative stress in all epidermal layers. Color code: black absence of oxidative stress, dark blue weak oxidative stress, green medium oxidative stress, yellow/white significant oxidative stress. BEMT bis-ethylhexyloxyphenol methoxyphenyl triazin, TDSA terephthalylidene dicamphor sulfonic acid

which included 45 or $75 \mathrm{~J} / \mathrm{cm}^{2}$ of UVA. These spectra are the mean of 15 scans and are given in adjusted normalized mean monochromatic absorption factor (mAF; lambda).

\section{Sunscreen J Prevents PLE}

Under "in-use" test conditions, under the South African sun, only one subject of the 41 participants had a notable reaction, which was diagnosed by a dermatologist as sunburn due to overexposure and/or poor application of the tested product. Sunscreen J was judged to be efficient in preventing skin reactions 


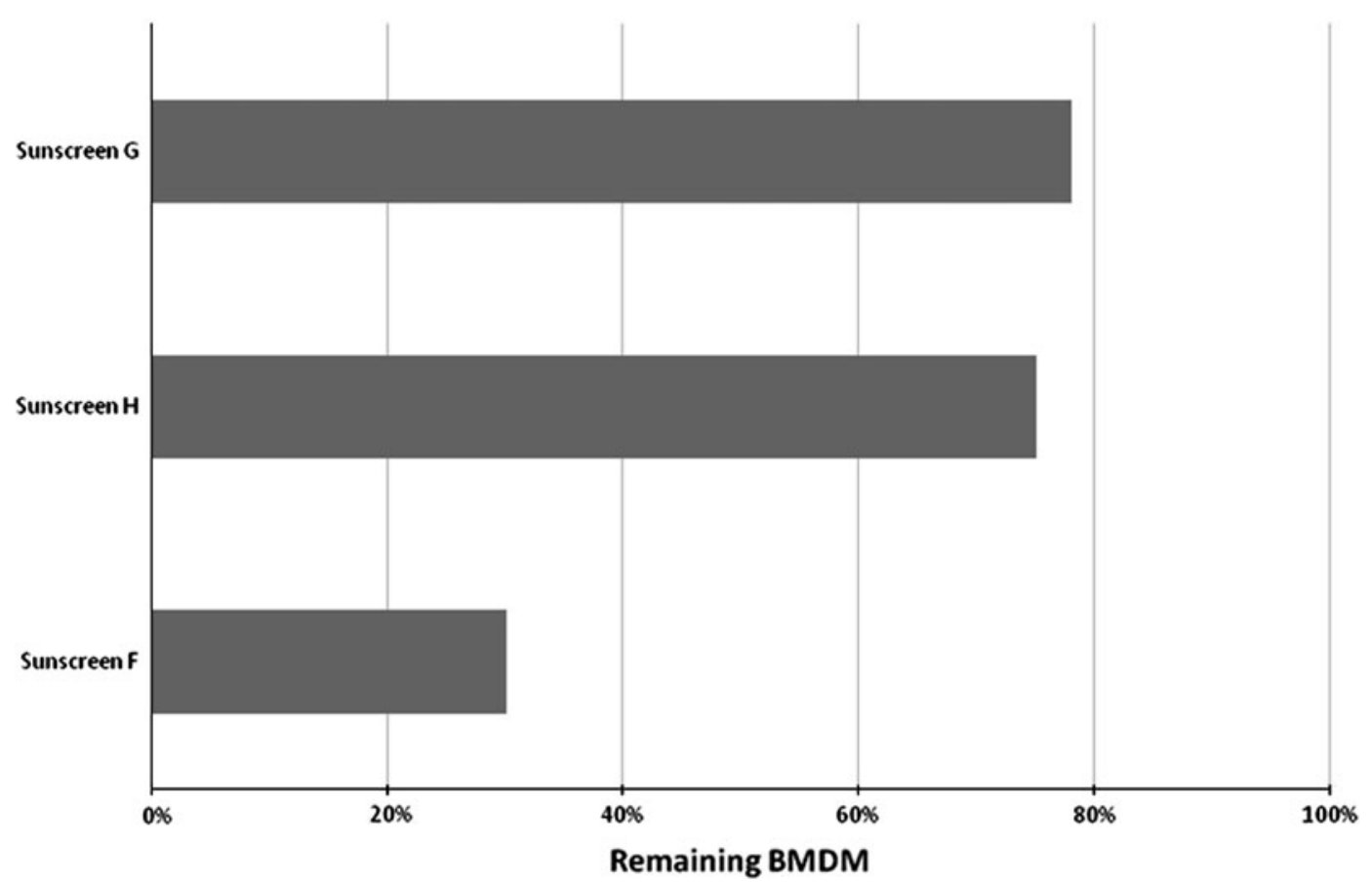

Fig. 4 Comparison of the remaining percentage of BMDM in Sunscreen F, G, and H evaluated by HPLC after $1 \mathrm{~h}$ SSR-exposure. BMDM is photostabilized by association with BEMT or ILS. BEMT bis-ethylhexyloxyphenol methoxyphenyl triazin, BMDM butyl methoxy dibenzoyl methane, HPLC high-performance liquid chromatography, $I L S$ isopropyl lauroyl sarcosinate, SSR solar simulated radiation

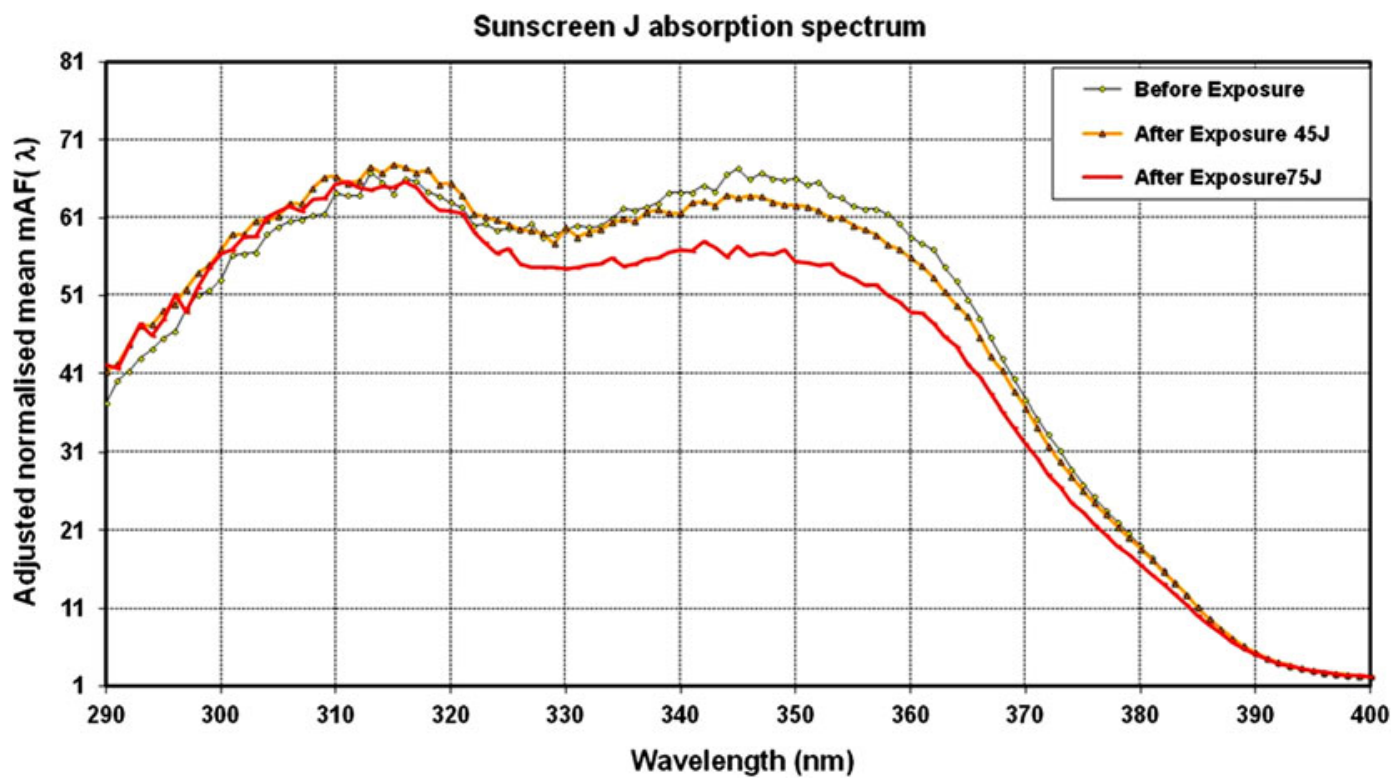

Fig. 5 UV absorption spectra of Sunscreen J before and after exposure to SSR doses, including either $45 \mathrm{or} 75 \mathrm{~J} / \mathrm{cm}^{2} \mathrm{UVA}$, and demonstrating the photostability of this formula. SSR solar simulated radiation 
(declarative judgment) associated with PLE by $85 \%$ of the volunteers. Sunscreen J was also highly appreciated for its cosmetic qualities (83\% of the volunteers) and its efficacy against sunburn (95\% of the volunteers).

\section{DISCUSSION}

Over the past 20 years, an increasing number of publications have reported the damaging effects of UVA radiation. It has been proven that UVA radiation induces molecular, cellular, and clinical damage, which may lead to photoinduced aging, immune system depression, altered gene expression, oncogenes, and tumor suppressor gene modulation partly responsible for skin cancer development [17].

In parallel to this increased knowledge, progress has been achieved in sunscreen technology. A variety of UVA filters are now available and the present authors have combined them with UVB filters to produce a high protection and photostability with a minimum concentration of active ingredients. However, further UVA filters could be investigated for their synergistic qualities. There remains a need to improve sun-care formulations, particularly to provide broad UVA protection without losing cosmetic properties. Additionally, further development of new filter combinations with a low environmental impact should be continued.

As knowledge increases in photochemistry and photobiology, formulators face many challenges when developing new sunscreens. Simply including UV filters in a formulation base does not ensure efficacy, photostability, or an aesthetically pleasing texture that is easy to apply, i.e., a product that the consumer will apply and continue to re-apply.

The fact that there is an increasing concern about the possible impact of chemical filters on the environment pushes formulators to try to reduce the amount of filters without decreasing the efficacy. This is an additional challenge.

To address these demands, a combination of UV filters was selected and was shown to be synergistic in terms of SPF and UVA-PF. In addition, the spectrofluorimetry measurements validated the performance of the TDSA and BEMT combination in reducing oxidative stress in both the stratum corneum and the living epidermis layers.

The ILS, an oily vehicle, was expected to improve the solubility of the lipophilic UV filters, as well as BMDM photostability. The authors successfully developed a new sunscreen formulation that included the filters TDSA, BEMT, and BMDM within the ILS vehicle. The present results clearly showed that this new association of filters with ILS oil technology obtained both high sunscreen photoprotective efficacy and photostability over the entire UV range.

In conclusion, the UVA filters, TDSA, BEMT, and BMDM, solubilized in the oily derivative of the ILS, and combined with an UVB filter (octocrylene) and titanium dioxide, produce a sunscreen with a high UVA-PF (UVA-PF 38) and a SPF $50+$. This product is photostable even after $>4 \mathrm{~h}$ exposure under zenithal sun. It prevents UV-induced biological damage as well as skin reactions of PLE.

\section{ACKNOWLEDGMENTS}

This study was fully financed by L'Oréal. The authors recognize the contribution of Florence L'Alloret, Didier Candau, and Martin Josso for their assistance in the technology conception and formulation. The authors also thank Lydie Gauchet, Ana-Maria Pena, Anne Colonna, Marie-Jocelyne Pygmalion, and Laetitia Ruiz 
for their role in the efficacy evaluations. The manuscript was written by Dr. Sophie Seité and Hélène Meaudre. Amy Whereat provided proofreading services. Dr. Seite is the guarantor for this article, and takes responsibility for the integrity of the paper as a whole.

Conflict of interest. All authors are employees of L'Oréal, France.

Open Access. This article is distributed under the terms of the Creative Commons Attribution Noncommercial License which permits any noncommercial use, distribution, and reproduction in any medium, provided the original author(s) and source are credited.

\section{REFERENCES}

1. Lim HW, Cooper K. The health impact of solar radiation and prevention strategies: report of the environment council, American Academy of Dermatology. J Am Acad Dermatol. 1999;4:81-99.

2. Bech-Thomsen N, Wulf HC. Sunbathers application of sunscreen is probably inadequate to obtain the sun protection factor assigned to the preparation. Photodermatol Photoimmunol Photomed. 1993;9: 242-4.

3. Wright MF, Wright ST, Wagner RF. Mechanisms of sunscreen failure. J Am Acad Dermatol. 2001;44: 781-4.

4. Naylor M, Farmer KC. The case for sunscreens: a review of their use in preventing actinic damage and neoplasia. Arch Dermatol. 1997;133:1146-54.

5. Schauder S, Ippen H. Contact and photocontact sensitivity to sunscreens: review of a 15-year experience and of the literature. Contact Dermatitis. 1997;37:221-32.

6. Darvay A, White IR, Rycroft JL, Jones AB, Hawk JL, McFadden JP. Photoallergic contact dermatitis is uncommon. Br J Dermatol. 2001;145:597-601.

7. Fourtanier A, Bernerd F, Bouillon C, Marot L, Moyal D, Seité S. Protection of skin biological targets by different types of sunscreens. Photodermatol Photoimmunol Photomed. 2006;22:22-32.

8. Commission of the European Communities. Commission recommendation of 22 September 2006 on the efficacy of sunscreen products and the claims made relating thereto. Official Journal of the European Union 2006/647/EC, L265/39-L265/ 43 (2006) Document number 32006H0647. Available at: http://eurlex.europa.eu/LexUriServ/ LexUriServ.do?uri=OJ:L:2006:265:0039:0043:EN:PDF. Accessed Mar 192012.

9. Forestier S. Rationale for sunscreen development. J Am Acad Dermatol. 2008;58(suppl. 2):S133-8.

10. Deflandre A, Lang G. Photostability assessment of sunscreens: benzylidene camphor and dibenzoylmethane derivatives. Int J Cosmet Sci. 1988;10:53-62.

11. Bonda CA. The photostability of organic sunscreen actives: a review. In: Shaath NA, editors. Sunscreen Regulations and Commercial Development. Sunscreens. 3rd edition. New York: Taylor and Francis; 2005. p. 321-49.

12. Herzog B, Werhrle M, Quass K. Photostability of UV absorber system in sunscreens. Photochem Photobiol. 2009;85:869-78.

13. Dencausse L, Galland A, Clamou JL, Basso J. Validation of HPLC method for quantitative determination of Tinosorb ${ }^{\circledR} S$ and three other sunscreens in a high protection cosmetic product. Int J Cosmet Sci. 2008;30:373-82.

14. International sun protection factor (SPF) test method CFFA, SA, Colipa, JCIA, CTFA 2006. Available at: http://www.cosmeticseurope.eu/ publications-cosmetics-europe-association/guidelines. html?view=item\&id=21. Accessed Mar 192012.

15. Japan Cosmetic Industry Association (JCIA). Measurements Standard for UVA Protection Efficacy. Tokyo: JCIA; 1996.

16. Rastegi RP, Singh SP, Hader DP, Sinha R. Detection of reactive oxygen species (ROS) by the oxidant sensing probe $2^{\prime}, 7^{\prime}$ dichlorodihydrofluorescein diacetate in the cyanobacterium Anabaema variabilis PCC 7937. Biochem Biophys Res Commun. 2010;397:603-7.

17. Seite S, Fourtanier A, Moyal D, Young AR. Photodamage to human skin by suberythemal exposure to solar ultraviolet radiation can be attenuated by sunscreens: a review. Br J Dermatol. 2010;163:903-14. 